\title{
Generality of the Kindling Phenomenon: Some Clinical Implications
}

\author{
J. P. J. PINEL AND P. H. VAN OOT
}

SUMMARY: The purpose of the present investigations was to explore the generality of the kindling phenomenon and its applicability to clinical situations. Whether local brain stimulation, electroconvulsive shock (ECS), or metrazol the consequence of periodic administration of convulsive agents was found to be the same; in each case repeated application of the agent resulted in the gradual development and intensification of convulsive symptoms (kindling). Moreover, in each case the resulting intensification was not specific to the agent being used and seemed to increase the responsiveness to convulsive agents in general. In the present studies this interaction was seen in the form of an intensified alcohol withdrawal syndrome observed 18 days after cessation of a series of metrazol injections, amygdaloid stimulations, or ECS. Thus, it appears that one of the hazards of the convalsive therapies is that they may induce enduring changes in brain function which leave the patient in a state of increased susceptibility to a variety of potentially convalsive agents.

RÉSUMÉ: Le but du présent travail est d'étudier la généralité du phénomène de "kindling" et son application aux situations cliniques. Dans tous les cas étudiés (stimulation cérébrale locale, électrochocs ou metrazol) le résultat fut le même: l'application répétée de l'agent résulte en un développement graduel et une intensification des symptômes convulsifs ("kindling"). Cette réponse n'est pas spécifique à l'agent employé qui augmente la réponse aux agents convulsifs en général. Ceci comporte un danger réel lors de traitements convulsifs repétés chez l'humain, quelque soit leur forme.

From the Department of Psychology, University of British Columbia, Vancouver, British Columbia.

Reprint requests to Dr. J. P. J. Pinel. Dept. of Psychology, University of British Columbia, Vancouver, B.C. V6T IW5 Canada.
Periodic bipolar stimulation of any of a number of sites throughout the olfactory-limbic system can lead to the gradual development and intensification of behavioral convulsions (kindling) in a variety of species (Goddard, McIntyre \& Leech, 1969) even at current intensities initially too low to produce any behavioral or electrographic effects. For example, if the rat amygdala is periodically stimulated at a level which produces neither electrographic nor behavioral effects, eventually the afterdischarge (AD) threshold may be reduced to the point that subsequent stimulations reliably elicit an $\mathrm{AD}$. If stimulations are then continued, mild motor automatisms may appear which increase in severity with each successive stimulation until motor seizures (MSs), characterized by facial and forelimb clonus, rearing, and a loss of equilibrium can be reliably elicited (Racine, 1972a).

Although kindling can be produced from a wide variety of olfactory, limbic, and cortical areas, the number of stimulations necessary to kindle full MSs is a function of the site of stimulation (Goddard, McIntyre \& Leech, 1969). However, since the amygdala has been found to be particularly responsive, most studies of the kindling phenomenon have employed amygdaloid stimulation. Regardless of the level of responsiveness of any particular structure, however, kindling cannot be produced unless stimulations are distributed over time. Goddard, McIntyre and Leech (1969) found the number of stimulations leading to kindled convulsions to be inversely related to the duration of the interval between stimulations; it was difficult, if not impossible, to kindle rats with interstimulation intervals of less than 20 min., and inter- stimulation intervals of $24 \mathrm{hr}$. or longer were found to be optimal. In a subsequent study using different stimulation parameters, Racine, Burnham, Gartner and Levitan (1973) confirmed the general inverse relation between the duration of the interstimulation intervals and the number of stimulations required to kindle full MSs but found that intervals as short as $1 \mathrm{hr}$. could be used without significantly retarding the rate of kindling. Moreover, they found that kindling and not just the expression of MSs was blocked at short intervals; massed stimulations (15 stimulations administered at 15-min. intervals) produced only a slight reduction in the number of distributed stimulations later required to kindle full MSs.

Racine (1972a) has provided convincing evidence that the elicitation of ADs at the site of stimulation is a necessary prerequisite for the kindling of MSs; periodic stimulation maintained at a level below the $\mathrm{AD}$ threshold did not lead to the development of MSs, nor did it reduce the number of suprathreshold stimulations later required for kindling. Moreover, the main electrophysiological correlate of the kindling process appears to be the degree to which the ADs generalize from the site of stimulation to other neural structures (Racine, Gartner \& Burnham, 1972); during the course of kindling, spike amplitudes in secondary foci progress from very small or nonexistent to amplitudes as great as those in the primary focus (Racine, 1972a). Racine (1972b) has also reported that an appreciable reduction in the AD threshold can occur during kindling but at least three lines of evidence suggest that the reduction in the AD threshold is independent of 
the kindling which can occur concomitantly. First, periodic amygdaloid stimulation maintained below the $A D$ threshold reduces the $A D$ threshold without leading to the development of MSs (Racine, 1972a); second, stimulation of posterior neocortex does not kindle MSs even though it reduces the AD threshold (Racine, 1975); and third, the reduction of the AD threshold appears to be restricted to the site of stimulation while kindling of one brain site greatly influences the degree to which other brain sites can be kindled (Goddard, McIntyre \& Leech, 1969; Racine, 1972b; Racine, 1975; McIntyre \& Goddard, 1973).

Perhaps the most important feature of the changes in neural function induced by periodic brain stimulation is that they are enduring if not altogether permanent. Goddard, McIntyre \& Leech (1969) rekindled rats after a 12-week stimulation-free period and found a saving of about $90 \%$ in the number of stimulations required to elicit a full MS. Similarly, Racine (1972b) found that the decreases in AD threshold produced in rats by repeated hippocampal or amygdaloid stimulation persisted at least 6 weeks after all stimulations were curtailed. Although the permanence of the changes in MSs are more striking and have thus been studied in more detail, the changes in AD threshold are particularly important since they can be produced by subthreshold stimulation. Even stimulation levels which produce no obvious electrographic or behavioural change can produce a lasting increase in the susceptibility of the brain to later stimulations.

\section{CLINICAL IMPLICATIONS} OF KINDLING RESEARCH

The purpose of the present paper is to discuss some of the clinical implications of recent research on the kindling phenomenon. Although investigations directed at revealing the mechanisms underlying the kindling phenomenon may ultimately provide valuable information concerning epileptic processes in particular and neural plasticity in general which will be of tremendous practical importance, a number of investigators including ourselves have directly approached the kindling phenomenon from a more applied perspective. At this time attempts to employ the kindling phenomenon to collect data with direct clinical implications appear to fall into two relatively distinct categories. In this section these two approaches will be discussed and a third method of using the kindling paradigm to gather information of clinical relevance will be presented.

The utility of the kindling phenomenon as a model of epileptogenesis was the first clinical application of the kindling paradigm to be emphasized (c.f. Goddard, McIntyre $\&$ Leech, 1969; Morrell, 1973). There are striking parallels between the kindling phenomenon and the progressive development of epileptic symptoms in human patients; in untreated clinical cases as in kindled animals there is often a progressive development of symptoms within a particular attack as well as from one fit to the next (Hughlings Jackson, 1870). However, until recently the kindling phenomenon has had one critical shortcoming as a model of epileptogenesis even though the kindling procedure has several important advantages over methods which have traditionally been used to induce epilepsy in experimental animals (Goddard, McIntyre \& Leech, 1969). By definition, human epilepsy is a spontaneously-recurring, selfsustained, paroxysmal dysfunction of the brain; but early efforts to kindle animals to the point where they would display such spontaneous seizures did not meet with success (Goddard, McIntyre \& Leech, 1969). Thus, the major shortcoming of kindling in rats as a model of epileptogenesis was that kindling did not seem capable of inducing a bona fide epileptic condition. However, recent experiments by Wada and his associates and studies from our own laboratory have clearly demonstrated that periodic amygdaloid stimulation will eventually result in the production of spontaneously recurring seizures in both cats (Wado \& Sato, 1973; Wada, Sato \& Corcoran,
1974) and rats (Pinel, Phillips, Mucha \& Deol, 1973; Pinel \& Rovner, in progress).

In the rat, producing spontaneous seizures appears to be simply a matter of extending the number of periodic brain stimulations. If amygdaloid stimulations are continued beyond the point where most kindling experiments are curtailed, there is a gradual reduction in the day-today variability of the class 5 MS pattern (Racine, 1972a) followed by the eventual development of two new MS patterns. The first involves multiple rearing and falling sequences and the second can best be described as a running fit. In the week or so before spontaneous seizures are observed, the response to stimulation becomes very erratic; sometimes the response to stimulation is greatly attenuated or even totally absent. However, since animals have not been monitored continuously, it is not clear whether this variable response is the antecedent or the consequence of the development of spontaneity. In our experiments spontaneity generally has developed after five months of $1 \mathrm{sec} ., 60 \mathrm{~Hz}, 400$ $\mu \mathrm{A}$ stimulations administered on a schedule of 12 to 16 per week.

On the basis of our studies and those of Wada and his associates it appears that the electrographic basis of the development of spontaneity is the same in both the rat and the cat. In both cases the development and proliferation of interictal spike discharges appears to be the major electrographic correlate of the epileptogenesis of spontaneous seizures.

Now that it has been well established that repeated local brain stimulation will eventually lead to the progressive development of a truly epileptic syndrome, the kindling model should prove to be a valuable and widely used tool in the controlled, experimental study of factors associated with epileptogenesis. We (Pinel \& Rovner) are currently collaborating with Madge and Arnold Scheibel of the UCLA School of Medicine on such a project designed to uncover some of the neuroanatomical correlates of stimulationinduced epileptogenesis. 
The second major clinical application of the kindling paradigm has been to use it as a procedure for assessing the convulsant and anticonvulsant properties of drugs and other stimuli. A good example of this approach is the recent study by Babington and Wedeking (1973) in which the effects of a variety of centrally-active agents on kindled seizures elicited from the amygdala or sensorimotor cortex of rats were studied. Once the seizures were stabilized (c.f. Pinel, Phillips \& Deol, 1974), drug effects on seizure duration were assessed. Antidepressive drugs were found to be more effective in suppressing seizures elicited by amygdaloid stimulation than those elicited from the cortex, whereas both antianxiety and antiepileptic drugs exerted nonselective blockade but the antianxiety agents were more potent. Neuroleptic drugs and stimulants produced minor inhibitory and facilitatory effects respectively. On the basis of their experience using kindled seizures for assessing pharmacological effects, Babington and Wedeking outlined a number of advantages of the kindling paradigm as a neuropharmacologic test system over more traditional alternatives, however they overlooked its most important feature. By using the kindling paradigm as an assay device the effects of drugs can be assessed not only on each individual seizure but on the progressive intensification of seizures from stimulation to stimulation. By determining the extent to which anticonvulsant drugs are capable of blocking the kindling MSs, it may eventually become possible to predict which anticonvulsants will be most successful when used prophylactically in a clinical situation (Ward, 1975). Moreover, such studies may provide insights into the neurochemical basis of the kindling phenomenon in particular and epileptogenesis in general.

The effects of pharmacologic agents on the kindling process have been studied by several investigators. Wise and Chinerman (1974), for example, reported that diazepam and phenobarbital but not diphenylhydantoin can block the progressive intensification of MSs but not the local decrease in AD threshold produced by repeated electrical stimulation of the amygdala. Similarly, Arnold, Racine and Wise (1973) have reported that the cholinergic blocking agent atropine retards the rate of MS development without influencing the local $\mathrm{AD}$ threshold, whereas attentuation of catecholaminergic activity by reserpine or 6-hydroxydopamine facilitated kindling.

The main shortcoming of attempts to study the effects of pharmacologic agents on the kindling process, at least when viewed from the clinical perspective, is that only the effects on the initial portion of the kindling process have been studied. Agents which have no effect on the genesis of stimulation-induced MSs may prove to be very effective in suppressing the eventual expression of spontaneous seizures.

We have recently begun to approach the clinical implications of the kindling phenomenon from a different perspective. Stimulated by the recent warning of Goddard (1971) we have tried to determine what implications the kindling phenomenon might have for the application of various forms of convulsive therapy in the treatment of psychopathology. In the following sections data will be reported that show that convulsive therapy may leave an individual in an enduring state of hypersusceptibility to a variety of convulsive agents.

\section{INTENSIFICA TION OF ALCOHOL WITHDRAWAL BY KINDLING}

Since the discovery that electrical stimulation of discrete sites of the brain can exert a powerful influence on behavior (Olds \& Milner, 1954), there has been growing interest in the possible therapeutic value of local brain stimulation in general and stimulation of the so-called "motivational" areas in particular. There have already been numerous reports of clinical applications of this technique. Electrical current has been applied to the human brain in an attempt to identify epileptic foci (Adams \& Rutkin, 1972), to treat schizophrenia
(Cerletti, 1956), to change the emotional level of psychotic patients (c.f. Heath, 1970), to treat homosexuality (Moan \& Heath, 1972), as a prosthesis (Brindley \& Lewin, 1971), and to relieve chronic pain (Heath \& Mickle, 1960). Recently, however, Goddard (1971) warned that if brain stimulation is employed for therapeutic purposes, there is a real danger that seizures will be elicited during treatment unless an effort is made beforehand to identify and use procedures that are not conducive to kindling.

The purpose of our first experiment in this series was to demonstrate a further hazard of inadvertent kindling. Can kindling leave an organism more susceptible to seizures produced by agents other than local brain stimulation? If this is the case, the main danger is not that a patient could suffer a few accidental seizures during the course of the treatment, but that the patient could be rendered irreversibly susceptible to convulsive agents administered long after therapy has been curtailed. We concentrated our study on one of the most prevalent convulsive agents in our society - alcohol. Alcohol itself is an anticonvulsant but once it has been metabolized it leaves the organism in a state of heightened seizure susceptibility (McQuarrie \& Fingl, 1958). In alcoholic individuals the withdrawal from alcohol often precipitates a withdrawal syndrome characterized by tremors and other "mild" epileptic symptoms, but in severe cases it can also lead to grand mal seizures and death (Itil, 1970). Even in organisms who have had little experience with alcohol and display no obvious alcohol withdrawal symptoms there is an increased susceptibility to seizures induced by other agents following alcohol withdrawal (McQuarrie \& Fingl, 1958). Can kindling increase the susceptibility of organisms to seizures produced by the subsequent withdrawal from alcohol?

Thirty male, black-hooded rats (Canadian Breeding Farms and Laboratories, LaPrairie, Quebec) were implanted with unilateral, bipolar electrodes aimed at the center of the amygdaloid complex and then a 


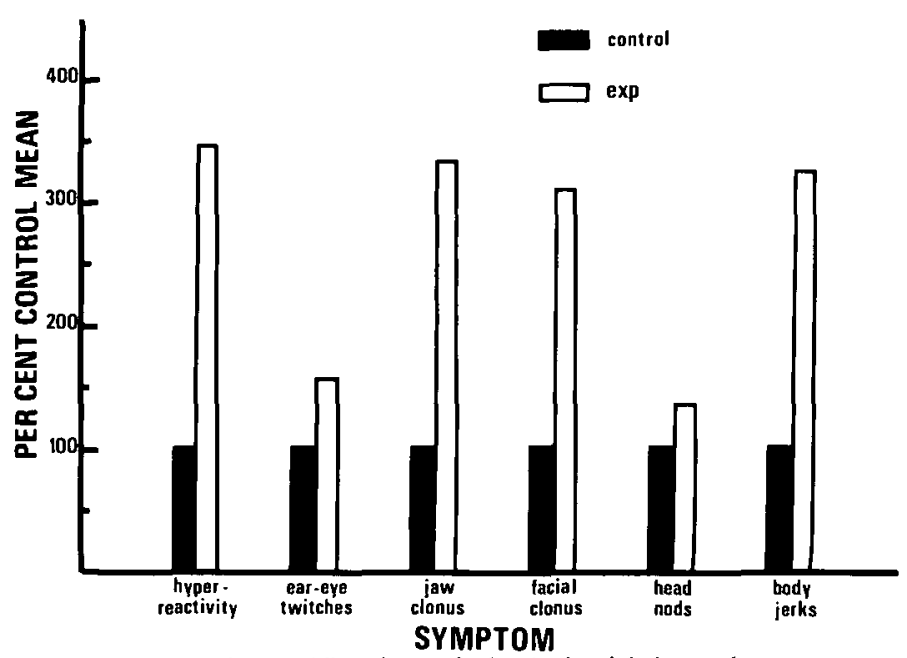

Figure 1-The intensification of alcohol withdrawal symptoms following electrical, amygdaloid kindling. The 45 stimulations were administered 3 per day, 5 days per week. The controls were implanted and treated as experimentals, except that they received no stimulation. Prior to withdrawal assessment all subjects received 45 ethanol intubations administered at 8-hr. intervals. The incidence of each symptom as presented in comparison to the control value arbitrarily presented as $100 \%$.

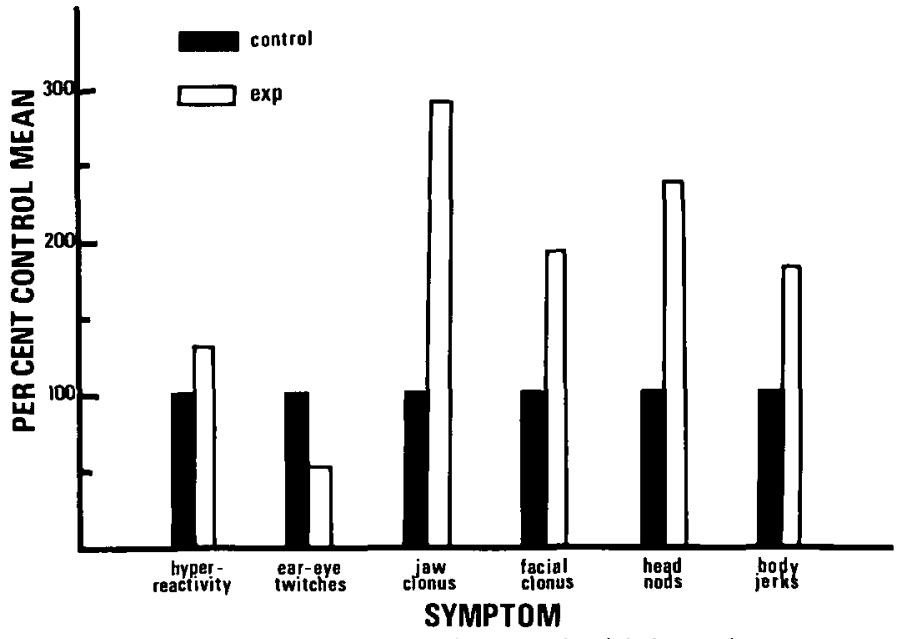

Figure 3-The intensification of alcohol withdrawal symptoms following repeated administrations of metrazol. The experimental subjects received 42 distributed metrazol injections, whereas the controls were injected with metrazol only twice. Prior to withdrawal assessment all subjects received 45 ethanol intubations administered at 8-hr. intervals. The incidence of each symptom is presented in comparison to an arbitrary control mean of $100 \%$. randomly selected group of 15 animals were kindled with 45,1 -sec., $400-\mu \mathrm{A}, 60-\mathrm{Hz}$ brain stimulations administered 3 times per day, 5 days per week. The remaining $15 \mathrm{im}$ planted animals served as unstimulated controls.

Three days after the last stimulation both groups of animals were subjected to 45 intubations of a $20 \%$ volumetrically prepared alcohol solu-

Figure 2-The development and increase in MS severity with repeated metrazol injections. The controls received metrazol on the first and last trials and saline on the intervening trials. The interval between injections was 3 days, with a 9-day, injection-free period preceding the last injection.

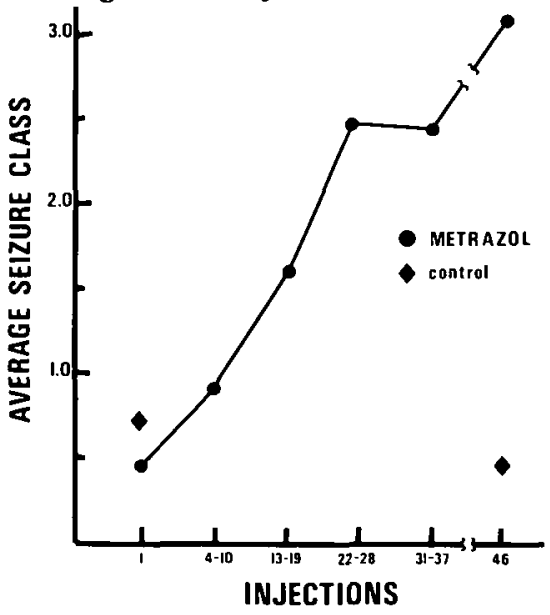

tion according to a procedure we have described elsewhere (Mucha, Pinel \& Van Oot, 1975). Withdrawal assessments commenced 9 hours after the last intubation and all assessments were performed by a researcher who did not know the experimental history of the animals. Each animal was placed under "formal" observation for 2-minute periods every 3 hours and the presence or absence of the following withdrawal symptoms was assessed: 1) rhythmic activity of the mouth, 2) facial tremors, 3) rhythmic eye or ear twitching, 4) myoclonic body jerks, 5) rhythmic head nodding, and 6) hyperreactivity to handling. Since there were six observation sessions, the maximum score an animal could receive for each of the six symptoms was 6 with an overall maximum score of 36 for all the symptoms.

It is apparent in Figure 1 that the withdrawal syndrome displayed by the kindled animals was much more severe than that of the controls. For the purpose of making an overall statistical comparison each animal was given a score for each of the six symptoms which was a percent of the mean score obtained for the unstimulated control animals for that symptom. Thus, the mean of the control animals for each symptom was $100 \%$ and the control mean for all six symptoms considered together was also $100 \%$. In contrast, the mean of these overall scores for the kindled animals was $249.8 \%\left(\mathrm{U}=16 ; \mathrm{N}_{1} / \mathrm{N}_{2}=11 / 11\right.$; $\mathrm{p}<.005$ one-tailed). Furthermore, three full clonic seizures like those seen in the advanced stages of amygdaloid kindling were observed during the course of withdrawal, all in experimental subjects.

A similar experiment was designed to determine whether subsequent alcohol withdrawal could be intensified by amygdaloid stimulation which was of insufficient intensity to elicit ADs and thus to kindle MSs. Eleven animals were implanted with electrodes as in Experiment 1 and an initial AD threshold was determined for each animal. Each subject was stimulated for $1 \mathrm{sec}$. at $25 \mu \mathrm{A}$, then, if no AD was elicited, at 50 , then 75 and so on at increments of $25 \mu$ A every $6 \mathrm{hr}$. until an AD was produced. The intensity which evoked an AD was considered the threshold for that animal. Each rat was stimulated until one $\mathrm{AD}$ was produced and then received control handling without stimulation until the thresholds of all subjects were determined. Subjects were then divided into two groups on the basis of these threshold scores. One group $(n=6 ; \bar{x}=131.2 \mu \mathrm{A})$ 
was then placed on a stimulation regimen similar to the schedule used in Experiment 1 with an initial stimulation level for each animal $15 \%$ below the threshold for that animal. Whenever an AD was produced in this series of stimulation sessions however, the intensity of subsequent stimulations was reduced by $15 \%$. In this way stimulation levels were kept below the AD threshold for the majority of stimulations. Thus, although the average threshold drop for the stimulated group was $62.2 \%$, no animals developed full MSs and only one animal displayed any overt epileptic symptoms in response to the stimulations. The remaining animals $(n=5 ; \bar{x}=134.4 \mu \mathrm{A})$, served as unstimulated controls.

After the administration of alcohol and assessment of the withdrawal reaction, the raw scores for each of the symptoms were again converted to a percentage of the control mean for respective symptoms. As in the first experiment each of the six withdrawal symptoms was observed significantly more frequently in the stimulated animals $(\bar{x}=197.1 \%)$ than in the unstimulated controls $(\overline{\mathrm{x}}=$ $100.0 \%)\left(\mathrm{U}=3 ; \mathrm{N}_{1} / \mathrm{N}_{2}=5 / 6 ; \mathrm{p}<.02\right)$.

The present data have revealed an important aspect of the kindling phenomenon. It is now clear that repeated, local brain stimulation does more than merely increase the susceptibility to seizures produced by subsequent brain stimulations. Repeated stimulation apparently alters the neural substrate in such a way that the organism becomes generally more susceptible to convulsive agents. In the present experiments this change was manifested in the form of an intensified alcohol withdrawal syndrome. The fact that stimulation either above or below the AD threshold effectively intensified the symptoms suggests that the reduction of the AD threshold was an important factor in the increased severity of the withdrawal syndrome. Although suprathreshold stimulation is required for the kindling of MSs, subas well as suprathreshold stimulations can effectively reduce the AD threshold (Racine, 1972a, b).

There are obvious parallels between the present results and a phenomenon which has been widely reported in the experimental epilepsy literature. Epileptic foci which are themselves not spontaneously active can be readily activated by the systemic administration of a convulsive agent, even at low doses. Since brain stimulation will eventually lead to a syndrome characterized by spontaneous seizures (Pinel, Mucha \& Phillips, 1975), the kindling procedure can be viewed as a controlled method of establishing a type of epileptic focus. In the present situation, although the genesis of the focus had not reached the point where MSs were spontaneously occurring, spontaneous interictal spiking was often recorded from the site of stimulation. Since this spiking was observed only in stimulated animals, it suggests that a focus was in fact being produced by the stimulation. It is not at all surprising, therefore, that these animals should show an increased epileptic response to a systemic convulsive agent such as alcohol withdrawal. Moreover, since a wide variety of systemic agents have been shown to interact with epileptic foci, there is no reason to believe that alcohol is the only agent which can interact with prior brain stimulation to intensify epileptic symptoms. Withdrawal from barbiturates and many of the non-barbiturate, sedative drugs and minor tranquilizers can produce convulsions similar to those seen in alcohol withdrawal (Itil, 1970). Conversely there are numerous drugs, including a number of the major tranquilizers and antidepressives as well as some of the hallucinogens, which directly increase the susceptibility to seizures (Itil, 1970). In fact, in a study recently completed in our laboratory (Pinel, Skelton \& Mucha, 1975) we have found that repeated amygdaloid stimulation, either below or above the AD threshold, intensifies subsequent convulsive reactions to metrazol in rats.

\section{KINDLING WITH OTHER AGENTS}

One of the main factors determining the degree to which studies of the kindling phenomenon can be applied to clinical situations, is the degree to which such effects can be produced by agents other than local brain stimulation. Although the demonstration that repeated amygdaloid stimulation can intensify subsequent alcohol withdrawal even when administered at subthreshold levels is of clinical importance, the applicability of these results is severely restricted by the fact that local brain stimulation is an infrequently employed, albeit interesting, therapeutic technique. Thus, after establishing the generality of the kindling phenomenon in one sense, that is by showing that amygdaloid kindling produces an enduring increase in responsiveness to a variety of convulsive agents, we approached the generality issue from another perspective. To what extent can kindling be produced by agents other than local brain stimulation?

Kindling-type effects have been reported in response to the repeated administration of a variety of convulsive agents. For example, Mason and Cooper (1972) administered "subconvulsive" doses of metrazol to rats at 3-day intervals. After a few injections minor myoclonic responses developed and eventually grand mal seizures were elicited in some animals. Leech (1970) observed that mice which are initially resistant to audiogenic seizures eventually became responsive to the stimulation if they were exposed to a short burst of audiogenic stimulation each day. At first the sound elicited little more than a startle response in most mice of "resistant" strains but after several days of exposure, fits of running and jumping were elicited and with a few additional stimulations grand mal convulsions were typically produced. Prichard, Gallagher and Glaser (1969) found that if seizures were elicited with flurothyl ether at a rate of not more than once a day there was a progressive reduction of seizure threshold in rats, mice, and guinea pigs. With each presentation of the flurothyl, there was a decline in the latency to the first myoclonic jerk and to sustained convulsion, and an increase in the severity of the MS pattern. Finally, Vosu and Wise (1975) have recently shown that carbachol injected daily into the amygdala, hippocampus, or caudate of rats 
at "subconvulsive" doses will eventually elicit MSs.

The effects of repeated electroconvulsive shock (ECS) were of particular interest, first because ECS is the most widely used form of convulsive therapy and second, because the effects of repeated ECS appeared to stand in apparent conflict with the effects produced by other convulsive agents; an increase rather than a decrease in convulsive threshold was the well-documented result of a series of ECSs. For example, Essig, Groce and Williamson (1961) found that a series of twice daily ECSs administered to cats produced an increase in seizure threshold which gradually returned to normal when the series was terminated; and an even greater increase in seizure threshold occurred when the ECSs were administered four times per day.

In our studies, however, we have found results consistent with the kindling notion (Ramer \& Pinel, 1974). In our first studies ECS was administered to rats once every three days at a constant intensity of either 15 or $25 \mathrm{ma}$. At both current intensities the result was the same; with each successive ECS there was a gradual intensification of the seizure pattern as reflected in declining latencies to forelimb and hindlimb extension and an increasing incidence of hindlimb extension. In other words, these results were in accord with previous studies of the kindling phenomenon but in apparent contradiction with the bulk of studies of repeated ECS. A careful examination of this literature suggested that the main factor responsible for this discrepancy was the duration of the intervals between ECS. In our initial experiment this interval was three days and in the only other studies in which increased seizure susceptibility following repeated ECS was reported (Zarrow, Pawlowski \& Denenberg, 1962; Pollack, Rosenthal \& Macey, 1963) the interval was a week. In all of the other studies ECS was administered at intervals of 24 $\mathrm{hr}$. or less and a progressively increasing seizure threshold was the result. The hypothesis that the interstimulation interval was the critical factor was confirmed by an experiment in which a 10-ma ECS was repeatedly administered to rats at 1-hr., 1-day, or 3-day intervals. The results were in complete accord with our hypothesis. When ECSs were administered at 3-day intervals, there was a progressive increase in seizure susceptibility as demonstrated by increasingly severe MS patterns and declining latencies to forelimb and hindlimb extension, thus confirming the results of Experiment 1. At the 1-hr. interval, however, the results were opposite; the MSs grew progressively less severe in terms of all three measures. The intermediate 1-day condition produced intermediate effects; there were no systematic changes in seizure severity.

This relationship between the duration of the interstimulation interval and changes in the susceptibility to ECS-produced seizures, is basically the same as that observed for other convulsive agents; facilitation occurs at only long intervals while inhibition is observed when intervals are short. This suggests that the mechanisms underlying the progressive facilitatory and inhibitory effects produced by the repeated administration of any of a variety of convulsive agents are basically the same. However, the exact form of the re- lationship between the interstimulation interval and systematic changes in seizure susceptibility depends on the agent employed and whether or not such agents are administered at sub- or suprathreshold levels.

\section{INTENSIFICATION OF}

ALCOHOL WITHDRAWAL BY

PERIODIC ECS OR METRAZOL

Our finding that repeated amygdaloid stimulation produces a general increase in the responsiveness to convulsive agents coupled with the observation that the periodic administration of any of a wide variety of convulsive agents can induce a lasting increase in seizure susceptibility suggested that convulsive agents which have been frequently administered to human patients for clinical purposes may be capable of inducing lasting increases in susceptibility to a wide variety of convulsive agents. We have studied this question in two separate but similar experiments. In one experiment 41 i.p. injections of metrazol were administered at three-day intervals to experimental rats; the control animals were injected with metrazol on the first day and with equal volumes of saline thereafter. In order to assess the permanence of the metrazol effects, an additional metrazol injec-

Figure 4-The increase in four measures of MS severity with repeated ECS at two current intensities with an interval between stimulations of three days. The first and last trials were conducted at $15 \mathrm{~mA}$ in both groups.

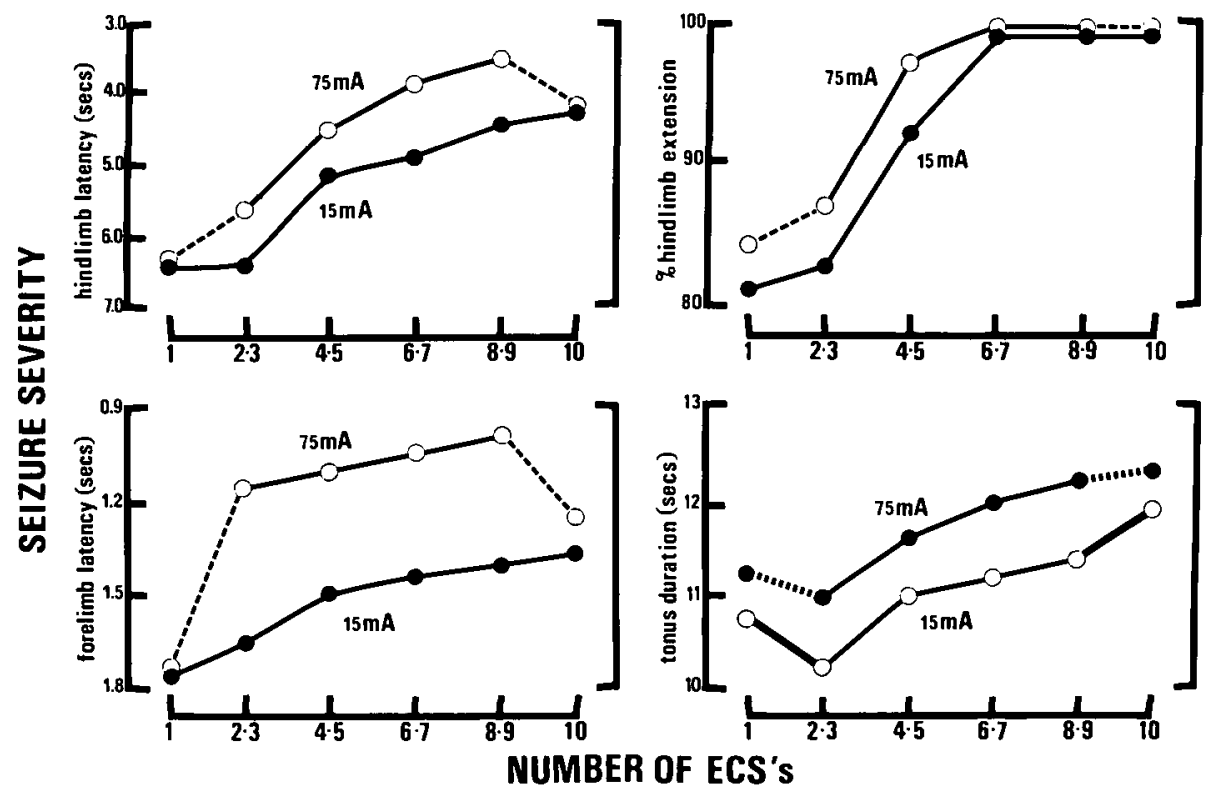




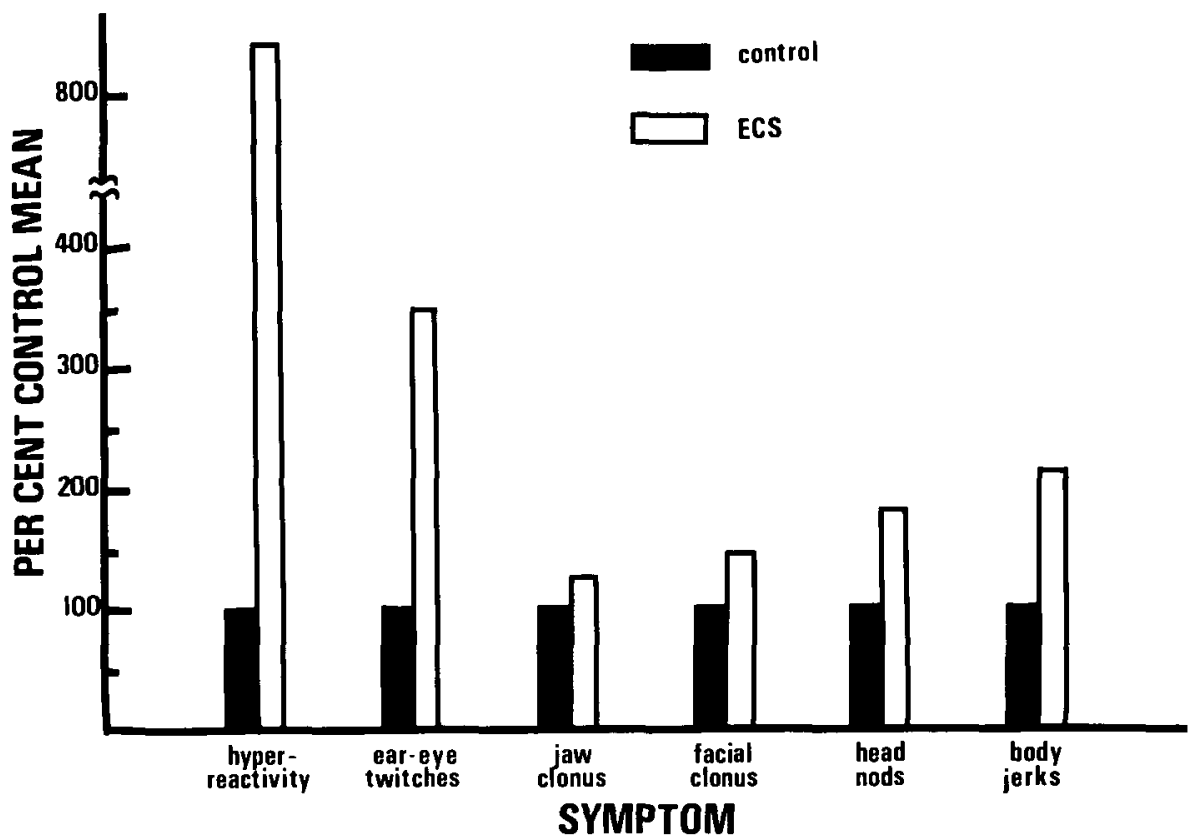

Figure 5-The intensification of alcohol withdrawal symptoms following repeated ECS administrations. The controls were implanted and handled as experimentals, but were not stimulated. Prior to withdrawal assessment all subjects received 45 ethanol intubations administered at 8 -hr. intervals. The incidence of each symptom is presented in comparison to an arbitrary control mean of $100 \%$.

tion was administered to all subjects 9 days after injection 41 . The initial metrazol injection produced no responses in about $70 \%$ of the experimental subjects while very mild facial tremors were noted in the remaining subjects. Inspection of Figure 2 confirms the previous report of Mason and Cooper (1972); with repeated administration of low doses of metrazol there was a gradual development and intensification of convulsive responses. Since the development of MSs in response to repeated metrazol injections was much the same as that observed in response to repeated limbic stimulation, we assessed the severity of the MSs using the 5-stage scale devised by Racine (1972a). The five stages are (1) facial movements only, (2) facial movements and head nodding, (3) facial movements, head nodding, and forelimb clonus, (4) facial movements, head nodding, forelimb clonus, and rearing (5) facial movements, head nodding, forelimb clonus, rearing, and falling.

As in our previous experiment both the experimental and control subjects were then intubated with alcohol every $8 \mathrm{hr}$. for a 2 -week period and the incidence of various withdrawal reactions was then assessed. In Figure 3 the incidence of each of the six withdrawal symptoms is presented in comparison to the arbitrary control standards of $100 \%$. Clearly the overall withdrawal reaction was much more severe in the experimental subjects $\left(U=10, N_{1} / N_{2}=4 / 15\right.$, $\mathrm{p}<.025$ one-tailed) even though the controls themselves had received two injections of metrazol during the course of the experiment.

Finally, in our most recent experiment, all experimental rats received 0.5 sec., 15-ma ECS administered through implanted skull screws, and the same ECS was again administered to all the experimental subjects 30 days later. In the intervening period all the experimental animals received 8 ECSs administered at 3 day intervals. For half of these animals the intensity of the intervening ECSs was 15 ma while the other half were stimulated at $75 \mathrm{ma}$. Control subjects were handled but remained unstimulated.

Figure 4 confirms the results of our previous studies (Ramer \& Pinel, 1974) and adds to their generality. The repeated administration of ECS at both intensities produced a gradual intensification of the convulsive response which was reflected in each of four separate measures of seizure severity. Although the rate of seizure intensification was the same at both current intensities the higher 75 ma current consistently evoked seizures of greater severity thus confirming the validity of each of our four measures.

In the second stage of this study both experimental and control subjects were subjected to the 15-day series of ethanol intubations followed by withdrawal assessment as in our previous studies. Since the intensity of the ECS had no appreciable effect on the incidence of the withdrawal symptoms the data of these two groups were combined for presentation in Figure 5. Again the facilitatory effect of repeated convulsive stimulation is clearly illustrated $(U=104$, $\mathrm{N}_{1} / \mathrm{N}_{2}=21 / 24, p<.001$ one-tailed).

\section{CONCLUSIONS}

Although the present studies have been presented from an applied perspective, they have served to illustrate two important features of the kindling effect which will have important consequences for attempts to uncover the basic nature of the kindling phenomenon. First, the present studies make it clear that the intensification of MSs produced by local electrical stimulation of responsive brain sites is but one manifestation of a much more general response. In the present studies, a progressive intensification of MSs was demonstrated in response to periodic ECS and injections of metrazol while other investigators have produced similar responses with the periodic administration of flurothyl vapour, audiogenic stimulation, or intracerebral injections of carbachol. Second, the intensification of MSs produced by the periodic administration of one agent is not restricted to MSs elicited by that agent. In the present experiments this generality was seen in the form of intensified alcohol withdrawal following periodic amygdaloid stimulation, periodic metrazol injections, or periodic ECS. Although the study of periodic, local, 
electrical brain stimulation may prove to be the most productive approach to studying this phenomenon, an explanation of the kindling phenomenon will ultimately have to account for its generality. Moreover, it may prove possible to gain some valuable insights into the kindling process by comparing the epileptogenesis induced by various agents.

The generality of the kindling phenomenon has two rather direct implications for the use of convulsive or potentially convulsive therapies. First, clinicians should be aware of the enduring changes in brain function which can be induced by such agents and weigh the hazards involved against possible therapeutic benefits. Second, if such therapeutic agents are employed, it is important that the subsequent exposure to potentially convulsive agents be carefully monitored. At present, convulsive therapies are commonly administered in conjunction with pharmacological agents which themselves can have convulsive effects (c.f. Itil, 1970).

Of particular importance was our finding that lasting increases in the responsiveness to convulsive agents can be induced by repeated amygdaloid stimulation administered below the AD threshold. Repeated brain stimulation may be dangerous even though neither MSs nor ADs are elicited during the course of treatment.

\section{DISCUSSION}

Dr. Wada: I regret that Dr. Mason is not here with us today. It is interesting that metrazol produces similar progressive reinforcement of seizure manifestations. We have attempted the same sort of thing but with a slightly different idea in that if we combined metrazol and photic stimulation application over a period of time we might be able to produce photosensitivity among non-sensitive Papio papio or totally non-epileptic rhesus monkeys. Drs. Osawa and Corcoran spent quite a bit of time on this and the results have been totally negative in spite of a prolonged effort. An interesting thing that happened is that one of the rhesus monkeys began to have spontaneous seizures. We still have this particular rhesus monkey with us and, as a matter of fact, Dr. Mizoguchi is kindling him. If you compare the metrazol-treated animals against non-treated rhesus monkey, there is no difference in terms of seizure development so far. I had anticipated that it might be different or faster. In order to look into this further, and knowing that a perinatal seizure often predisposes one to later development of temporal lobe epilepsy, we started subjecting kittens in the perinatal period to repetitive seizures. We have waited for about 1.5 years now and we are just about to start to kindle them hoping to learn the consequence of early metrazol seizure on kindling. On the other hand, we have been using the kindling model for examination of the prophylactic property of a number of drugs, dilantin, phenobarb, carbamazepine, delta-8, delta 9-THC and taurine. Among al these agents we find that phenobarbitol and carbamazepine as well as delta 9-THC are very effective prophylactic agents whereas DPH is not. This is very interesting in view of Drs. Rapport and Ojemann's work using a cobalt model with a focus in the frontal area. They found DPH was most effective in their preparation. It is quite conceivable that if we used frontal kindling, the results could have been different. It is obvious that we need to examine more than one area to gain a comprehensive view of any drug's prophylactic potency preferably in several species. Dr. Livingston or Dr. Racine may have something to add to this later on. I would now like to call upon Dr. Corcoran to give us a brief summary of what he has observed with taurine and delta 8- and 9. THC. Dr. Corcoran: There has been a lot of recent interest in taurine as an anti-epileptic agent based mainly on Van Gelder's work. We did, I guess, the obvious experiment of giving taurine to amygdala kindled cats, rats and baboons. As it turned out, it had absolutely no effect on their seizure pattern although it is capable of wiping out photically-induced seizures in photosensitive baboons. It has no effect on kindled amygdaloid seizures in the same animal. Dr. Racine tells me that he has done the same thing in rats but he has looked at the effects on development of seizures as well. Again, taurine is entirely negative in terms of prophylaxis. Dr. Pincl: You know that Dr. Gaito at York is also doing that? Dr. Corcoran: Yes. Gaito's results sound somewhat different. He is trying to claim that there is some effect on the very early manifestations of kindled amygdaloid seizures in rats. We will have to see what he says in Quebec City. This, of course, brings up one of the values of kindling that you mentioned and that is that you can look at drugs that aren't established anti-epileptic agents and try to get some profile of their effect. As Dr. Wada mentioned we've looked at delta 8and delta 8- tetrahydro-cannabinol which are the major psychoactive ingredients of marijuana. We found that delta 8-, which is present only in trace amounts in cannabis, has some effect on established kindled seizures in rats and baboons and it has some prophylactic effect in the development of kindled amygdaloid seizures in rats but it's not a genuine prophylactic effect. At least, not under the circumstances in our experimental situation. The animals, when the drug is withdrawn and they are rekindled, show immediate seizures even though they never expressed them during kindling which validates Racine's point about using that test of rekindling when you are working at prophylactic effects. Delta 9 in rats is the same. It has a prophylactic effect which under our circumstances is not a genuine effect. However, with cats in amygdaloid kindling, Dr. Wake showed delta 9-exerts a genuine prophylactic effect. The ani- mals are entirely protected from the effects of amygdaloid stimulation. It's as if they're naive when they're retested without the drug. so that I think that it will be interesting to use this preparation to compare the well known anti-epileptics with these other possibly useful therapeutic drugs. Dr. Pinel: One thing that I might add is that $I$ think it is very important in these studies to carry them all the way through to spontaneity. That is, you might find that certain drugs will have certain effects on the early stages of kindling but may have different effects on the expression of spontaneity. With the rats it would be tremendously difficult because of the amount of time involved but I think this may be an extremely important aspect of these kinds of experiments. Dr. Corcoran: I have a question regarding your report of these more advanced stages of seizure development in rats past the "classical" C5 stage. I think part of these results are undoubtedly due to prolonging the kindling and taking the animals out beyond the appearance of the first seizures, but I think that there is also the possibility that there is at least an interaction with the current level used to produce the seizures. We find that with $60 \mathrm{~Hz}$ sine wave the threshold for afterdischarge in the lateral amygdala in rats is roughly $30-40 \mu$ a for one sec of stimulation. and that is the whole order of magnitude below the current levels you have been using. I think we haven't carried the animals as far as you have in time, so that I don't know whether or not our animals would develop these manifestations. We see the running fits and so on but mostly in animals that have been pre-treated with 6-hydroxydopamine. I' $m$ wondering if you think it is possible that this interaction does exist and that the current level is an important variable as well. $D r$. Pincl: I sort of have the hunch that with lower current levels we might have an easier time. We find a general inverse relationship in animals where we have observed any kind of damage, usually from infection around the electrode tip. These are the animals that don't become spontaneous; I don't think that there is much of an effect. Dr. Wada: Since the following three papers are dealing with the same topic of frontal lobe and kindling, I would like to suggest that discussion on these papers begin at the end of the third paper. Hopefully, this will enable us to have more time for the general discussion at the end.

\section{ACKNOWLEDGEMENTS}

The authors would like to extend their thanks to D. Ramer, R. F. Mucha, L. I. Rovner, and R. Skelton for their efforts in collecting data reported in this paper.

Data discussed in this paper were collected under the support of grants from the National Research Council, Medical Research Council, and Non-Medical Use of Drugs Directorate awarded to the senior author.

\section{REFERENCES}

ADAMS, J. E. and RUTKIN, B. B. (1972). Diagnostic telemetry from depth electrodes in epilepsy. Confinia Neurologica, 34, 363.

ARNOLD, P. S., RACINE, R. J. and WISE, R. A. (1973). Effects of atropine, 
reserpine, 6-hydroxydopamine, and handling on seizure development in the rat. Experimental Neurology, 40, 457.

BABINGTON, R. G. and WEDEKING, P. W. (1973). The pharmacology of seizures induced by sensitization with low intensity brain stimulation. Pharmacology Biochemistry \& Behavior, 1, 461.

BRINDLEY, S. and LEWIN, W. S. (1971). The sensations produced by electrical stimulation of the visual cortex. In: Visual Prosthesis: The Interdisciplinary Dialog, edited by T. D. Sterling, E. A. Bering, S. V. Pollack and H. G. Vaughn. New York, Academic Press.

CERLETTI, I. (1956). Electroshock therapy. In: The Great Psychodynamic Therapies, edited by F. Marti-Ibaneg, A. M. Sackler, M. D. Sackler, and R. R. Sackler. New York, Hoeber-Harper.

ESSIG, C. F., GROCE, M. E. and WILLIAMSON, E. L. (1961). Reversible elevation of electroconvulsive threshold and occurrence of spontaneous convulsions upon repeated electrical stimulation of the cat brain. Experimental Neurology, 4, 37.

GODDARD, G. V., McINTYRE, D. C. and LEECH, C. K. (1969). A permanent change in brain function resulting from daily electrical stimulation. Experimental Neurology, 25, 295.

GODDARD, G. V. (1971). The Kindling Effect: A precautionary note on the therapeutic applications of localized brain stimulation. In: Neuroelectric Research, edited by D. V. Reynold and A. E. Sjoberg. Springfield, Illinois, C. C. Thomas.

HEATH, R. G. (1970). Perspectives for biological psychiatry. Biological Psychiatry, 2, 81.

HEATH, R. G. and MICKLE, W. A. (1960) Evaluation of seven years' experience with depth electrode studies in human patients. In: Electrical Studies on the Unanesthetized Brain, edited by E. R. Ramey and D. S O'Doherty. New York, P. B. Hoeber.

ITIL, T. M. (1970). Convulsive and anticonvulsive properties of neuropsychopharmaca. In: Modern Problems of Pharmacopsychiatry: Epilepsy (Vol. 4), edited by F. A. Freyhan, N. Petrilowitsch and P. Pichot. New York, S. Karger.

JACKSON, J. H. (1870). A study of convulsions. In: Selected Writings of John Hughlings Jackson (Vol. 1), edited by J. Taylor. New York, Basic Books.

LEECH, C. K. (1971). Sound-induced kindling resulting from daily bursts of loud noise presented to seven strains of mouse. Paper presented at Canadian Psychological Association meeting. St. John's, Newfoundland.
MASON, C. R. and COOPER, R. M. (1972). A permanent change in convulsive threshold in normal and brain damaged rats with small repeated doses of pentylene-tetrazol. Epilepsia, 13, 663.

McINTYRE, D. C. and GODDARD, G. V. (1973). Transference, interference and spontaneous recovery of convulsions kindled from the rat amygdala. Electroencephalography \& Clinical Neurophysiology, 35, 533 .

McQUARRIE, D. G. and FINGL, E. (1958). Effect of single doses and chronic administration of ethanol on experimental seizures in mice. Journal of Pharmacology and Experimental Therapeutics, 124, 264 .

MOAN, C. E. and HEATH, R. G. (1972). Septal stimulation and the initiation of heterosexual behavior in a homosexual male. Behavior Therapy and Experimental Psychiatry, 3, 23.

MORRELL, F. (1973). Goddard's kindling phenomenon: a new model of the "mirror focus". In: Chemical Modulation of Brain Function, edited by H. C. Sabelli. New York, Raven Press.

MUCHA, R. F., PINEL, J. P. J. and VAN OOT. P. H. (1975). Simple method for producing an alcohol withdrawal syndrome in rats. Physiology Biochemistry and Behavior, in press.

OLDS, J. and MILNER, P. (1954). Positive reinforcement produced by electrical stimulation of septal area and other regions of rat brain. Journal of Comparative and Physiological Psychology, 47, 419.

PINEL, J. P. J., MUCHA, R. F. and PHILLIPS, A. G. (1975). Spontaneous seizures generated in rats by kindling: a preliminary report. Physiological Psychology, in press.

PINEL, J. P. J., PHILLIPS, A. G. and DEOL, G. S. (1974). Effects of current intensity on kindled motor seizure activity in rats. Behavioral Biology, 11, 56.

PINEL, J. P. J.. PHILLIPS, A. G., MUCHA, R. F. and DEOL, G. S. (1973). Spontaneous seizures produced by long term repetitive amygdaloid stimulation in rats. Paper presented at IIIrd Annual Meeting of Society for Neurosciences.

PINEL. J. P. J., SKELTON, R. and MUCHA, R. F. (1975). Effects of current intensity on afterdischarge threshold during kindling. Paper presented at Canadian Psychological Association. Quebec City.

PINEL, J. P. J., VAN OOT, P. H. and MUCHA, R. F. (1975). Intensification of the alcohol withdrawal syndrome by repeated brain stimulation. Nature, 254, 510 .

POLLACK, M., ROSENTHAL, F. and MACEY, R. (1963). Changes in electroshock convulsive response with repeated seizures. Experimental Neurology, 7, 98.
PRICHARD, J. W., GALLAGHER, B, B. and GLASER, G. H. (1969). Experimental seizure threshold testing with fluorthyl. Journal of Pharmacology and Experimental Therapy, 166, 170.

RACINE. R. J. (1972b). Modification of seizure activity by electrical stimulation: $\mathrm{I}$. Afterdischarge threshold. Electroencephalography and Clinical Neurophysiology, 32 , 269.

RACINE, R. J. (1972a). Modification of seizure activity by electrical stimulation: II. Motor seizures. Electroencephalography and Clinical Neurophysiology, 32, 281.

RACINE, R. J. (1975). Modification of seizure activity by electrical stimulation: cortical areas. Electroencephalography and Clinical Neurophysiology, 38, 1.

RACINE, R. J. BURNHAM, W. M., GART NER, J. G. and LEVITAN, D. (1973). Rates of motor seizure development in rats sub. jected to electrical brain stimulation: strain and interstimulation interval effects. Electroencephalography and Clinical Neurophysiology, 35, 553.

RACINE, R. J., GARTNER, J. G. and BURNHAM, W. M. (1972). Epileptiform activity and neural plasticity in limbic structures. Brain Research, 47, 262.

RAMER, D. and PINEL, J. P. J. (1974). Kindling effect and ECS-induced seizures in rats. Paper presented at Canadian Psychological Association meeting, Windsor, Ontario.

VOSU, H. and WISE, R. A. (1975). Cholinergic seizure kindling in the rat: comparison of caudate, amygdala and hippocampus. Behavior Biology, 13, in press.

WADA, J. A. and SATO, M. (1973). Recurrent spontaneous epileptic seizure state induced by localized electrical stimulation. Neurology, 23, 447.

WADA, J. A., SATO, M. and CORCORAN, M. E. (1974). Persistant susceptibility and recurrent spontaneous seizures in kindled cats. Epilepsia, 15, 465.

WARD, A. A. (1975). Theoretical basis for surgical therapy of epilepsy. In: Advances in Neurology, Vol. 8: Neurosurgical Management of the Epilepsies, edited by D. P. Purpura, J. K. Penry, and R. D. Walter. New York, Raven Press.

WISE, R. A. and CHINERMAN, J. (1974). Effects of diazepam and phenobarbital on electrically-induced amygdaloid seizures and seizure development. Experimental Neurology, 45, 355.

ZARROW, M. S., PAWLOWSKI, A. A. and DENENBERG, V. H. (1962). Electroshock convulsion threshold in rats after alcohol consumption. American Journal Physiology, 203, 197. 